\title{
Sampling Bounds for 2-D Vector Field Tomography
}

\author{
Archontis Giannakidis • Maria Petrou
}

Published online: 24 March 2010

(C) Springer Science+Business Media, LLC 2010

\begin{abstract}
The tomographic mapping of a 2-D vector field from line-integral data in the discrete domain requires the uniform sampling of the continuous Radon domain parameter space. In this paper we use sampling theory and derive limits for the sampling steps of the Radon parameters, so that no information is lost. It is shown that if $\Delta x$ is the sampling interval of the reconstruction region and $x_{\max }$ is the maximum value of domain parameter $x$, the steps one should use to sample Radon parameters $\rho$ and $\theta$ should be: $\Delta \rho \leq \Delta x / \sqrt{2}$ and $\Delta \theta \leq \Delta x /\left((\sqrt{2}+2)\left|x_{\max }\right|\right)$. Experiments show that when the proposed sampling bounds are violated, the reconstruction accuracy of the vector field deteriorates. We further demonstrate that the employment of a scanning geometry that satisfies the proposed sampling requirements also increases the resilience to noise.
\end{abstract}

Keywords Vector field tomography · Radon transform . Sampling theorem $\cdot$ Scanning geometry $\cdot$ Inverse problems

\section{Introduction}

The reconstruction of 2-D vector fields from projections has opened new possibilities in a wide variety of disciplines. Applications that have been considered include:

\footnotetext{
A. Giannakidis

Faculty of Engineering and Physical Sciences, University

of Surrey, Guildford GU2 7XH, UK

e-mail: a.giannakidis@surrey.ac.uk

M. Petrou ( $\varangle)$

Department of Electrical and Electronic Engineering, Imperial

College, London SW7 2AZ, UK

e-mail: maria.petrou@imperial.ac.uk
}

blood flow imaging in vessels $[14,31]$; flow velocity imaging with MRI [22]; fluid mesoscale velocity imaging in ocean acoustic tomography [11, 16, 24]; fluid-flow imaging [3, 12, 15, 17-19, 32, 33]; electric field imaging in Kerr materials $[1,10,34]$; imaging of the component of the gradient of the refractive index field, which is transversal to the beam, in Schlieren tomography [3]; velocity field imaging of heavy particles in plasma physics [8]; density imaging in supersonic expansions and flames in beam deflection optical tomography [9]; non-destructive stress distribution imaging of transparent specimens in photoelasticity $[2,30]$; determination of temperature distributions and velocity vector fields in furnaces [26] and magnetic field imaging in tokomak in polarimetric tomography [29].

The mathematical basis for dealing with the problem of vector field tomographic mapping is formed by a projection transformation, the vectorial Radon transform. When we try to investigate planar vector fields in bounded domains, two classes of the vectorial Radon transform, that model the tomographic measurements, arise, depending on the interaction between the obtained measurements and the investigated vector field. The first type of the line integral transform, $J_{1}$, is

$J_{1}=\int_{L} \overline{\mathbf{f}}(x, y) \cdot \hat{\mathbf{s}} \mathrm{d} s=\int_{L} f_{\|} \mathrm{d} s$

where $\overline{\mathbf{f}}(x, y)$ is the planar vector field under investigation, $\hat{\mathbf{s}}$ is the unit vector along the integration (measurement) line $L$, $\mathrm{d} s$ is an element of path length along this line, $\cdot$ is the symbol for the dot product of two vectors and $f_{\|}$is the component of $\overline{\mathbf{f}}(x, y)$ along $L$. From (1), we may deduce that only the component of $\overline{\mathbf{f}}(x, y)$ along the line $L$ is observed in this type of measurement (longitudinal measurements). The second class of line integral transforms, $J_{2}$, is used to model tomographic measurements that collect information from the 
component of the investigated vector field perpendicular to the measurement line (transversal measurements):

$$
J_{2}=\int_{L} \overline{\mathbf{f}}(x, y) \cdot \hat{\boldsymbol{\rho}} \mathrm{d} s=\int_{L} f_{\perp} \mathrm{d} s
$$

Here $\hat{\boldsymbol{\rho}}$ is the unit vector perpendicular to the line of integration $L$, and $f_{\perp}$ is the component of $\overline{\mathbf{f}}(x, y)$ transverse to $L$. The integral transforms of (1) and (2) (which are the two types of vectorial Radon transform for planar vector fields) are natural generalisations of the classical Radon transform to vector fields.

When the inverse problem in 2-D vector field tomography was typically discussed in continuum terms, using a scalar Radon-based approach and relying only on lineintegral measurements, it turned out that the problem was under-determined [3, 13, 18, 30]. In particular, it was found that only one component of the vector field could be recovered from tomographic measurements. The recovered component was either the curl-free (irrotational) part or the divergence-free (solenoidal) part, depending on the physical principle of the measurements, namely the relation between the obtained set of measurements and the investigated vector field. An algebraic reconstruction method of this type, where the authors considered the problem of only reconstructing the solenoidal component from the tomographic data, was developed in [7].

One possible solution to this problem would be to collect data using both types of relation between the measurements and the examined vector field for every application. Indeed, such an amount of information would be sufficient to allow for a full reconstruction of the vector field as Braun and Hauck demonstrated in [3]. Unfortunately, there are only very few specialised applications (mainly in optics), where it is physically realizable to have all these measurements available. Moreover, as Norton showed in [18], we may have a full reconstruction based only on longitudinal measurements, as long as, apart from the longitudinal measurements, supplementary information about the vector field, especially boundary conditions and a priori information about the source distribution, is available as well. A study, where the developed algebraic methodology is about reconstructing a divergence-free vector field based only on longitudinal measurements, is presented in [25]. In [5] it was shown that the reconstruction problem may be solved if one relies not only on the integral measurements, but also on information concerning the attenuation distribution of the field.

In addition, Rouseff and Winters showed in [23] that a 2-D vector field reconstruction based on boundary data is possible for scattering geometries. However, the model they used for the available scattering measurements was different from the integral transforms of (1) and (2). An important paper, laying down the foundations for collecting measurements from any direction (i.e., integrating along any direction) to allow 3D MR flow velocity imaging, by using an
MR scanner and manipulating appropriately the gradients, is the work presented in [22].

It has recently been demonstrated [21] that in the discrete domain, the complete recovery ${ }^{1}$ of a $2-D$ band-limited vector field based only on a limited number of line-integral data may be achieved.

The reconstruction method described in [21] is based on linear algebra and casts the tomographic vector field reconstruction problem as the solution of a system of linear equations. Solving, however, the problem in the discrete domain requires the sampling of the continuous Radon domain parameter space. The variables of the parameter space are the radial and angular parameters used to define the scanning lines, over which we integrate to obtain the line-integral measurements. In this paper we consider vector field tomography, and by using the frequency properties of the vectorial Radon transform, we derive the lower bounds that must be imposed on the sampling rates of the variables in the parameter domain.

Sampling issues in relation to vector field tomography were also discussed in [7]. However, the authors of [7] considered the problem of only reconstructing one of the two components of the examined vector field from tomographic data, in line with the conclusions drawn in $[3,13,18,30]$. Their key insight was to extend efficient sampling schemes of scalar tomography for vector field tomography. They did not attempt to perform complete reconstruction of the examined vector field, but seemed merely interested in recovering only one component. In this paper we deal with the problem of reconstructing both components of a 2-D vector field based only on line-integral data and, therefore, we investigate sampling issues of the scanning geometry with a view to solving this problem.

This paper is organised as follows. In Sect. 2 we formulate the problem, set up our notation and present a brief summary of the algebraic reconstruction method presented in [21]. In Sect. 3 we derive the minimum sampling rates for the complete 2-D vector field reconstruction by using sampling theory for deterministic band-limited signals and the sinc-expansion procedure. In Sect. 4 we present some reconstruction results of static electric fields and demonstrate the effect of the sampling rate on the quality of reconstruction. In Sect. 5 we examine the effect of the sampling rate on resilience to noise. We conclude in Sect. 6.

\section{The Reconstruction Methodology}

In this section, we review the linear algebra-based reconstruction methodology that is presented in [21]. Let us assume that we have the digitised square 2-D domain that is

\footnotetext{
${ }^{1}$ Complete recovery here means the recovery of all components of a vector field at a finite number of sampling points of its domain.
} 


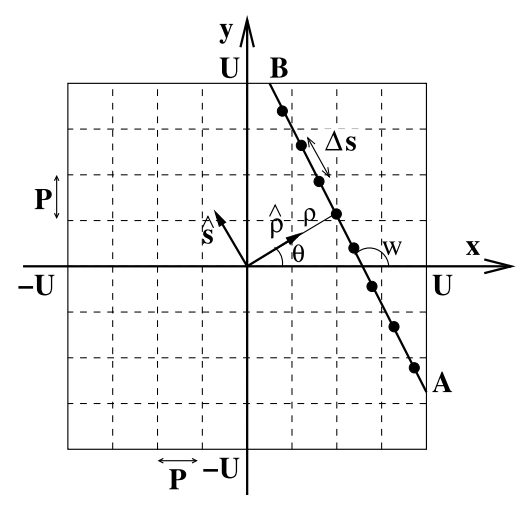

Fig. 1 A scanning line $A B$ that goes through the square digitised reconstruction region of size $2 U$. The line segment is sampled with sampling step $\Delta s$. The angle between the line segment and the positive direction of the $x$-axis is $w$. The size of the tiles, with which we sample the 2-D space, is $P \times P$. Also shown are the two parameters $\rho$ and $\theta$ used to define the scanning line (Radon domain coordinates) and the unit vectors $\hat{\mathbf{s}}$ and $\hat{\boldsymbol{\rho}}$ which are parallel and perpendicular, respectively, to line segment $A B$

shown in Fig. 1, within which we want to recover the vector field. The length of each side of the square domain is taken to be equal to $2 U$ and the origin of the axes of the coordinate system is chosen to be at the centre of the domain. The square domain is divided into tiles of finite size, $P \times P$, so that $K \equiv 2 U / P$ is an integer. The goal is to recover vector field $\overline{\mathbf{f}}(x, y)=f_{x}(x, y) \hat{\mathbf{x}}+f_{y}(x, y) \hat{\mathbf{y}}$ at the centre of every tile of this space, namely the sampling points of the domain.

A set of scanning lines $A B$ are considered, that are parameterised by parameters $\rho$ and $\theta$, where $\rho$ is the length of the normal to the scanning line, and $\theta$ is the angle this normal forms with the positive $x$ semi-axis (see Fig. 1). Each scanning line $(\rho, \theta)$ is described by

$\rho=x \cos \theta+y \sin \theta$

and yields a line-integral measurement of the projection of the vector field along or perpendicular to the line's direction.

By assuming that a pair of sensors measures only the integral of the component of the vector field along the scanning line $(\rho, \theta)$, then, the integral transform that models the process of data acquisition is given by (1). In order to translate into the digital domain, the integration expressed by (1) in the continuous domain, the integral of the vector field along the scanning line has to be expressed in terms of the components of the field at the sampling points of the 2-D grid. To do that, the scanning line is sampled with step $\Delta s$ (see Fig. 1), and the value of the vector field assigned to each centre of segment of length $\Delta s$ is the unknown value of the field at the nearest neighbour sampling point of the reconstruction domain. Along each segment of length $\Delta s$, the vector field is assumed constant, equal to the assigned value of the vector field at the corresponding sampling point of the line. It is possible, then, to approximate the integral of (1) by a sum by projecting the value of the field at each sampling point $l$ of the line onto the vector that represents the direction of the line:

$J_{i}=\sum_{l} \overline{\mathbf{f}}_{l} \cdot \overline{\Delta \mathbf{s}}$

Here $\overline{\mathbf{f}}_{l}=\left(f_{x_{l}}, f_{y_{l}}\right)$ are the unknown vector field values at sampling points $l$ and $\overline{\Delta \mathbf{s}}=\Delta s \hat{\mathbf{s}}=\Delta s(\cos w \hat{\mathbf{x}}+\sin w \hat{\mathbf{y}})$, where $w$ is the angle between the scanning line and the positive direction of the $x$-axis (see Fig. 1). The number of equations (4), we have, depends on the number of scanning lines we consider. In general, it is an over-determined system of linear equations, and its solution is obtained in the least-square error sense.

According to the theory of the Radon transform [6], a necessary requirement for producing accurate reconstructions is to sample uniformly the $(\rho, \theta)$ parameter space. In this paper we derive the minimum sampling rates that should be used for these parameters.

\section{Sampling the Vectorial Radon Transform}

In order to impose upper bounds on sampling intervals $\Delta \rho$ and $\Delta \theta$, we use sampling theory for deterministic bandlimited signals [20]. The derivation we provide is based on the sinc-expansion procedure [20] and the study of the 2-D frequency content of the available integral measurements in 2 -D vector field tomography.

Let us define a coordinate system $(\hat{\boldsymbol{\rho}}, \hat{\mathbf{s}})$, such that $\hat{\boldsymbol{\rho}}$ is the unit vector along the direction of the normal to a scanning line, and $\hat{\mathbf{s}}$ is the unit vector orthogonal to that, forming a right-handed coordinate system (see Fig. 1). The transformation relationships between this and the $(\hat{\mathbf{x}}, \hat{\mathbf{y}})$ coordinate systems, shown in Fig. 1, are:

$x=-s \sin \theta+\rho \cos \theta$

$y=s \cos \theta+\rho \sin \theta$

Also, by examining Fig. 1, we can see that the unit vector $\hat{\mathbf{s}}$, parallel to scanning line $A B$, may be written as:

$\hat{\mathbf{s}}=-\sin \theta \hat{\mathbf{x}}+\cos \theta \hat{\mathbf{y}}$

By combining the $(\rho, \theta)$ line parameterisation for scanning line $A B$, shown in Fig. 1, with (5)-(7), and assuming that $\overline{\mathbf{f}}(x, y)=\overline{\mathbf{0}}$ outside the square measurement region, (1), that describes the available data, becomes:

$$
\begin{aligned}
J_{i}(\rho, \theta)= & \int_{-\infty}^{+\infty} \overline{\mathbf{f}}(\rho \cos \theta-s \sin \theta, \rho \sin \theta+s \cos \theta) \\
& \cdot(-\sin \theta \hat{\mathbf{x}}+\cos \theta \hat{\mathbf{x}}) \mathrm{d} s
\end{aligned}
$$


If we assume that the unknown vector field $\overline{\mathbf{f}}(x, y)$ is bandlimited to a known upper limit spatial frequency ${ }^{2}$ and the sampling of the reconstruction region was made according to the Whittaker-Shannon theorem [20], then, vector field $\overline{\mathbf{f}}(x, y)$ may be recovered from the digital vector $\overline{\mathbf{f}}(m, n)=$ $f_{x}(m, n) \hat{\mathbf{x}}+f_{y}(m, n) \hat{\mathbf{y}}$ by convolution with a sinc function [20]

$$
\begin{aligned}
\overline{\mathbf{f}}(x, y)= & \sum_{m=0}^{M-1} \sum_{n=0}^{N-1} \overline{\mathbf{f}}(m, n) \frac{\sin \left(\frac{\pi}{\Delta x}\left(x-x_{m}\right)\right)}{\frac{\pi}{\Delta x}\left(x-x_{m}\right)} \\
& \times \frac{\sin \left(\frac{\pi}{\Delta y}\left(y-y_{n}\right)\right)}{\frac{\pi}{\Delta y}\left(y-y_{n}\right)}
\end{aligned}
$$

where $M$ and $N$ are the total numbers of samples in the $x$ and $y$ directions, respectively, $\Delta x$ and $\Delta y$ are the sampling intervals of the reconstruction domain, and $\left(x_{m}, y_{n}\right)$ are the coordinates of the vector field reconstruction points. ${ }^{3}$ By substituting (9) into (8), we obtain:

$$
\begin{aligned}
& J_{i}(\rho, \theta) \\
& =\int_{-\infty}^{+\infty} \sum_{m=0}^{M-1} \sum_{n=0}^{N-1} f_{x}(m, n) \\
& \quad \times \frac{\sin \left(\frac{\pi}{\Delta x}\left(\rho \cos \theta-s \sin \theta-x_{m}\right)\right)}{\frac{\pi}{\Delta x}\left(\rho \cos \theta-s \sin \theta-x_{m}\right)} \\
& \quad \times \frac{\sin \left(\frac{\pi}{\Delta y}\left(\rho \sin \theta+s \cos \theta-y_{n}\right)\right)}{\frac{\pi}{\Delta y}\left(\rho \sin \theta+s \cos \theta-y_{n}\right)}(-\sin \theta) \mathrm{d} s \\
& +\int_{-\infty}^{+\infty} \sum_{m=0}^{M-1} \sum_{n=0}^{N-1} f_{y}(m, n) \\
& \quad \times \frac{\sin \left(\frac{\pi}{\Delta x}\left(\rho \cos \theta-s \sin \theta-x_{m}\right)\right)}{\frac{\pi}{\Delta x}\left(\rho \cos \theta-s \sin \theta-x_{m}\right)} \\
& \quad \times \frac{\sin \left(\frac{\pi}{\Delta y}\left(\rho \sin \theta+s \cos \theta-y_{n}\right)\right)}{\frac{\pi}{\Delta y}\left(\rho \sin \theta+s \cos \theta-y_{n}\right)}(\cos \theta) \mathrm{d} s
\end{aligned}
$$

We want to study the sampling properties in the $(\rho, \theta)$ parameter domain. These are determined by the upper limit frequency of $J_{i}(\rho, \theta)$, as it is expressed by (10). However, some formulae are more easily derived at the $(p, \tau)$ domain,

\footnotetext{
${ }^{2}$ A gross estimate of the upper limit spatial frequency of the vector field we wish to recover is expected to be known. See, for example, [31] for blood flow imaging.

${ }^{3}$ It is not quite true that $\overline{\mathbf{f}}(x, y)$ is exactly recovered from $\overline{\mathbf{f}}(m, n)$ because the summations in (9), in principle, should be infinitely long, but these extra $\overline{\mathbf{f}}(m, n)$ are assumed to have zero value.
}

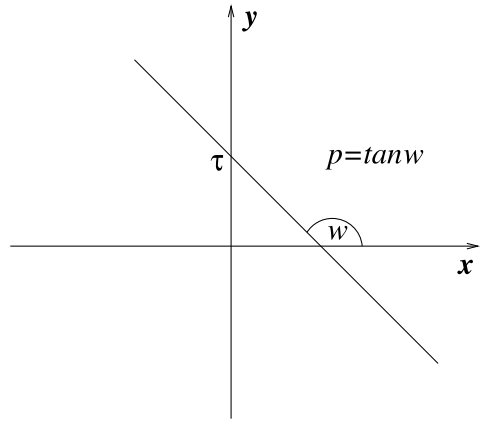

Fig. 2 The two parameters used to define a scanning line: slope $p$ and intercept $\tau$

where lines are parameterised by slope $p$ and intercept $\tau$ (see Fig. 2) as:

$y=p x+\tau$

The conclusions drawn can be easily translated, afterwards, to the $(\rho, \theta)$ domain. By using the $(p, \tau)$ line parameterisation described by (11), and, also, by taking into account that $\mathrm{d} x=-\sin \theta \mathrm{d} s, \mathrm{~d} y=\cos \theta \mathrm{d} s$ (see (5)-(6)) and $\mathrm{d} y=p \mathrm{~d} x$ (see (11)), (10) may be put in the form

$$
\begin{aligned}
J_{i}(p, \tau)= & \sum_{m=0}^{M-1} \sum_{n=0}^{N-1} f_{x}(m, n) I\left(p, \tau, x_{m}, y_{n}\right) \\
& +\sum_{m=0}^{M-1} \sum_{n=0}^{N-1} f_{y}(m, n) p I\left(p, \tau, x_{m}, y_{n}\right)
\end{aligned}
$$

where:

$$
\begin{aligned}
I\left(p, \tau, x_{m}, y_{n}\right)= & \int_{-\infty}^{+\infty} \frac{\sin \left(\frac{\pi}{\Delta x}\left(x-x_{m}\right)\right)}{\frac{\pi}{\Delta x}\left(x-x_{m}\right)} \\
& \times \frac{\sin \left(\frac{\pi}{\Delta y}\left(p x+\tau-y_{n}\right)\right)}{\frac{\pi}{\Delta y}\left(p x+\tau-y_{n}\right)} \mathrm{d} x
\end{aligned}
$$

In this analysis we deal with the reconstruction of a vector field only inside a rectangle. Hence, we choose to have $\Delta x=\Delta y$, so that all reconstruction sampling points lie in symmetrical intervals around the origin of the coordinate system. By taking this into account and also introducing variables

$t \equiv \frac{\pi}{\Delta x}\left(x-x_{m}\right)$

$\gamma \equiv \frac{\pi}{\Delta x}\left(p x_{m}+\tau-y_{n}\right)$

integral $I\left(p, \tau, x_{m}, y_{n}\right)$ is simplified to:

$I\left(p, \tau, x_{m}, y_{n}\right)=\frac{\Delta x}{\pi} \int_{-\infty}^{+\infty} \frac{\sin t}{t} \frac{\sin (p t+\gamma)}{p t+\gamma} \mathrm{d} t$ 
In order to calculate integral $I\left(p, \tau, x_{m}, y_{n}\right)$, we define two functions $g_{1}(\tau), g_{2}(\tau)$ as:

$g_{1}(\tau) \equiv \frac{\sin \tau}{\tau}$

$g_{2}(\tau) \equiv \frac{\sin (p \tau+\gamma)}{p \tau+\gamma}$

The convolution of these two functions gives:

$$
\begin{aligned}
& g(\tau) \equiv g_{2}(\tau) * g_{1}(\tau)=\int_{-\infty}^{+\infty} g_{2}(t) g_{1}(\tau-t) \mathrm{d} t \Longrightarrow \\
& g(\tau)=\int_{-\infty}^{+\infty} \frac{\sin (\tau-t)}{\tau-t} \frac{\sin (p t+\gamma)}{p t+\gamma} \mathrm{d} t
\end{aligned}
$$

By substituting $\tau=0$ in (19) we have:

$$
\begin{aligned}
& g(0)=\int_{-\infty}^{+\infty} \frac{\sin (-t)}{-t} \frac{\sin (p t+\gamma)}{p t+\gamma} \mathrm{d} t \Longrightarrow \\
& g(0)=\int_{-\infty}^{+\infty} \frac{\sin (t)}{t} \frac{\sin (p t+\gamma)}{p t+\gamma} \mathrm{d} t
\end{aligned}
$$

Hence, by (16) and (20), in order to compute integral $I\left(p, \tau, x_{m}, y_{n}\right)$, it is enough to compute the convolution described by (19) at $\tau=0$. However, convolution in the $\tau$ domain means multiplication in the frequency domain. The Fourier transform of function $g(\tau)$ is given by

$$
G(f)=G_{1}(f) G_{2}(f)
$$

where $G_{1}(f)$ and $G_{2}(f)$ are the Fourier transforms of $g_{1}(\tau)$ and $g_{2}(\tau)$, respectively. These are both sinc functions. Hence, their Fourier transforms are

$$
\begin{aligned}
& G_{1}(f)=\pi \operatorname{rect}(\pi f) \\
& G_{2}(f)=\frac{\pi}{|p|} \operatorname{rect}\left(\frac{\pi f}{|p|}\right) \exp \left(j 2 \pi f \frac{\gamma}{p}\right)
\end{aligned}
$$

where $\operatorname{rect}(f)$ is a rectangular function of value 1 for argument between $-\frac{1}{2}$ and $\frac{1}{2}$, and zero otherwise. The combination of (21)-(23) yields:

$G(f)=\frac{\pi^{2}}{|p|} \mathrm{W}(f) \exp \left(j 2 \pi f \frac{\gamma}{p}\right)$

where $\mathrm{W}(f)$ is similar to $\operatorname{rect}(\pi f)$ if $|p| \geq 1$, and similar to $\operatorname{rect}\left(\frac{\pi f}{|p|}\right)$ otherwise. The inverse Fourier transform of function $G(f)$ yields convolution function $g(\tau)$ :

$g(\tau)=\int_{-\infty}^{+\infty} G(f) \exp (j 2 \pi f \tau) \mathrm{d} f$

Two cases have to be distinguished: $|p| \geq 1$ and $|p|<1$.
1. Case $|p| \geq 1$. In this case, $\mathrm{W}(f)$ is similar to $\operatorname{rect}(\pi f)$ and (25) becomes:

$$
\begin{aligned}
g(\tau) & =\int_{-\frac{1}{2 \pi}}^{+\frac{1}{2 \pi}} \frac{\pi^{2}}{|p|} \exp \left(j 2 \pi f \frac{\gamma}{p}\right) \exp (j 2 \pi f \tau) \mathrm{d} f \\
& =\frac{\pi^{2}}{|p|} \int_{-\frac{1}{2 \pi}}^{+\frac{1}{2 \pi}} \exp \left(j 2 \pi f\left(\tau+\frac{\gamma}{p}\right)\right) \mathrm{d} f \\
& =\frac{\pi}{|p|} \frac{\sin \left(\tau+\frac{\gamma}{p}\right)}{\tau+\frac{\gamma}{p}}
\end{aligned}
$$

For $\tau=0$ we obtain:

$$
g(0)=\frac{\pi}{|p|} \frac{\sin \left(\frac{\gamma}{p}\right)}{\frac{\gamma}{p}}=\pi \frac{\sin \left(\frac{\gamma}{|p|}\right)}{\gamma}
$$

By comparing (16), (20) and (27), (16) becomes:

$$
I\left(p, \tau, x_{m}, y_{n}\right)=\Delta x \frac{\sin \left(\frac{\gamma}{|p|}\right)}{\gamma}
$$

2. Case $|p|<1$. In this case, $\mathrm{W}(f)$ is similar to $\operatorname{rect}\left(\frac{\pi f}{|p|}\right)$ and (25) becomes:

$$
\begin{aligned}
g(\tau) & =\int_{-\frac{|p|}{2 \pi}}^{+\frac{|p|}{2 \pi}} \frac{\pi^{2}}{|p|} \exp \left(j 2 \pi f \frac{\gamma}{p}\right) \exp (j 2 \pi f \tau) \mathrm{d} f \\
& =\frac{\pi^{2}}{|p|} \int_{-\frac{|p|}{2 \pi}}^{+\frac{|p|}{2 \pi}} \exp \left(j 2 \pi f\left(\tau+\frac{\gamma}{p}\right)\right) \mathrm{d} f \\
& =\frac{\pi}{|p|} \frac{\sin \left(|p|\left(\tau+\frac{\gamma}{p}\right)\right)}{\tau+\frac{\gamma}{p}}
\end{aligned}
$$

For $\tau=0$ we obtain:

$$
g(0)=\frac{\pi}{|p|} \frac{\sin \left(|p| \frac{\gamma}{p}\right)}{\frac{\gamma}{p}}=\pi \frac{\sin \gamma}{\gamma}
$$

By comparing (16), (20) and (30), (16) becomes:

$$
I\left(p, \tau, x_{m}, y_{n}\right)=\Delta x \frac{\sin \gamma}{\gamma}
$$

By taking (28) and (31) into account, (12) may be rewritten as:

$J_{i}(p, \tau)$

$$
\begin{aligned}
= & \underbrace{\sum_{m=0}^{M-1} \sum_{n=0}^{N-1} f_{x}(m, n) \Delta x \frac{\sin \left(\gamma \min \left\{1, \frac{1}{|p|}\right\}\right)}{\gamma}}_{C} \\
& +\underbrace{\sum_{m=0}^{M-1} \sum_{n=0}^{N-1} f_{y}(m, n) \Delta x \frac{p \sin \left(\gamma \min \left\{1, \frac{1}{|p|}\right\}\right)}{\gamma}}_{D}
\end{aligned}
$$


This expression of tomographic data in 2-D vector field tomography, obtained above, will be now used to establish the sampling requirements in the $(\rho, \theta)$ domain. The result of (32) shows that the continuous line-integral data are given by the sum of two quantities, $C$ and $D$.

By using lines with slope $|p| \leq 1$, the frequency content of quantity $C$ is determined by function $\frac{\sin \gamma}{\gamma}$, which, as a function of $\gamma$, has an upper limit frequency of $\frac{1}{2 \pi}$.

In a similar way, the frequency content of quantity $D$ is determined by function $\frac{p \sin \gamma}{\gamma}$, where $\frac{\sin \gamma}{\gamma}$ is a function of variables $p$ and $\tau$ (see (15)). Therefore, we may write:

$h(p, \tau) \equiv \frac{\sin \gamma}{\gamma}$

In order to determine the frequency content of the product $\frac{p \sin \gamma}{\gamma}=p h(p, \tau)$, we consider the 2-D FT of $h(p, \tau)$ :

$\tilde{h}\left(k_{p}, k_{\tau}\right)=\int_{-\infty}^{+\infty} \int_{-\infty}^{+\infty} h(p, \tau) e^{-j\left(k_{p} p+k_{\tau} \tau\right)} \mathrm{d} p \mathrm{~d} \tau$

By differentiating (33) with respect to $k_{p}$, we obtain:

$$
\begin{aligned}
& \frac{\partial \tilde{h}\left(k_{p}, k_{\tau}\right)}{\partial k_{p}} \\
& \quad=-j \int_{-\infty}^{+\infty} \int_{-\infty}^{+\infty} p h(p, \tau) e^{-j\left(k_{p} p+k_{\tau} \tau\right)} \mathrm{d} p \mathrm{~d} \tau \Longrightarrow \\
& j \frac{\partial \tilde{h}\left(k_{p}, k_{\tau}\right)}{\partial k_{p}}=\int_{-\infty}^{+\infty} \int_{-\infty}^{+\infty} p h(p, \tau) e^{-j\left(k_{p} p+k_{\tau} \tau\right)} \mathrm{d} p \mathrm{~d} \tau
\end{aligned}
$$

By examining (34), we notice that the second part of this equation gives the Fourier transform of the product $p h(p, \tau)$. Therefore, by taking into account the same equation, we may deduce that the product $p h(p, \tau)$ is band-limited to the upper limit frequency of function $h(p, \tau)$. Hence, we conclude that the frequency content of $D$ is also determined by function $\frac{\sin \gamma}{\gamma}$, and the overall tomographic data have, as a function of $\gamma$, an upper limit frequency of $\frac{1}{2 \pi}$. Thus, if $\gamma$ should be sampled, this should be done with a rate faster than $\pi$ :

$\Delta \gamma \leq \pi$

If we use the transformation relationships between the $(p, \tau)$ and $(\rho, \theta)$ domains (see (3) and (11))

$p=-\cot \theta$

$\tau=\frac{\rho}{\sin \theta}$

then, (15) becomes:

$\gamma=\frac{\pi}{\Delta x}\left(-\cot \theta x_{m}+\frac{\rho}{\sin \theta}-y_{n}\right)$
From (35) and (38) we obtain:

$\Delta \gamma=\left|\frac{\partial \gamma}{\partial \rho}\right| \Delta \rho=\frac{\pi}{\Delta x|\sin \theta|} \Delta \rho \leq \pi \quad \Longrightarrow$

$\Delta \rho \leq \Delta x|\sin \theta|$

Since we are examining lines with $|p| \leq 1$, hence, $|\cot \theta| \leq 1$. Therefore, $\theta \in\left[\frac{\pi}{4}, \frac{3 \pi}{4}\right] \cup\left[\frac{5 \pi}{4}, \frac{7 \pi}{4}\right]$ and $|\sin \theta|$, as a function of $\theta$, has values between 1 and $\frac{1}{\sqrt{2}}$. By using the minimum value of $\frac{1}{\sqrt{2}},(39)$ yields:

$\Delta \rho \leq \frac{\Delta x}{\sqrt{2}}$

Similarly, (35) and (38) give for the angular parameter:

$\Delta \gamma=\left|\frac{\partial \gamma}{\partial \theta}\right| \Delta \theta=\frac{\pi}{\Delta x}\left|\frac{x_{m}}{\sin ^{2} \theta}-\frac{\rho \cos \theta}{\sin ^{2} \theta}\right| \Delta \theta \leq \pi \quad \Longrightarrow$

$\frac{\pi}{\Delta x}\left|\frac{x_{m}-\rho \cos \theta}{\sin ^{2} \theta}\right| \Delta \theta \leq \pi \quad \Longrightarrow$

$\Delta \theta \leq \Delta x \underbrace{\left|\frac{\sin ^{2} \theta}{x_{m}-\rho \cos \theta}\right|}_{F}$

We want the sampling criterion that we shall derive to be valid for all values of $x_{m}$. Hence, we must find the minimum value of quantity $F$ in (41). For the denominator we have

$\left|x_{m}-\rho \cos \theta\right| \leq\left|x_{m}\right|+|\rho \cos \theta| \leq\left|x_{\max }\right|+\left|\rho_{\max } \cos \theta\right|$

where $x_{\max }$ and $\rho_{\max }$ are the maximum values of parameters $x$ and $\rho$, respectively. We consider uniform sampling in $(\rho, \theta)$ for the employed square domain of Fig. 1. Therefore, it must be $\rho_{\max } \leq x_{\max }$. Otherwise, for $\rho_{\max }>x_{\max }$ and $\theta=k \frac{\pi}{2}$ where $k$ is an integer, the resulting scanning lines do not lie within the region of interest. So, (42) becomes:

$\left|x_{m}-\rho \cos \theta\right| \leq\left|x_{\max }\right|(1+|\cos \theta|)$

Quantity $F$ in (41) becomes minimum when the numerator of $F$ becomes minimum and the denominator of $F$ becomes maximum. However, by taking into account (43) and the considered area of values of $\theta$, then, we may say that $F$ becomes minimum for $|\sin \theta|=|\cos \theta|=\frac{1}{\sqrt{2}}$. Hence, (41) may be written as:

$\Delta \theta \leq \Delta x \frac{\left(\frac{1}{\sqrt{2}}\right)^{2}}{\left|x_{\max }\right|\left(1+\frac{1}{\sqrt{2}}\right)} \Longrightarrow$

$\Delta \theta \leq \frac{\Delta x}{\sqrt{2}(1+\sqrt{2})\left|x_{\max }\right|}$ 
It must be noted that the evaluation of the upper bounds of (40) and (44) was very conservative in order to make sure that these expressions are valid for all values of $\left(x_{m}, y_{n}\right)$.

It can be easily proven that the same bounds for the sampling steps in the $(\rho, \theta)$ domain, as those expressed by (40) and (44), are obtained when using lines with slope $|p|>1$. In this case, it is easier to describe lines as $x=r y+\eta$ with $r=\frac{1}{p}$ and $\eta=-\frac{\tau}{p}$ and, then, obtain the sampling steps in the $(\rho, \theta)$ domain, based on the frequency properties of the parameter domain $(r, \eta)$.

In summary, if $\Delta x$ is the sampling interval of the reconstruction region and $x_{\max }$ is the maximum value of parameter $x$, the steps one should use to sample parameters $\rho$ and $\theta$ should be:

$$
\begin{aligned}
& \Delta \rho \leq \frac{\Delta x}{\sqrt{2}} \\
& \Delta \theta \leq \frac{\Delta x}{\sqrt{2}(1+\sqrt{2})\left|x_{\max }\right|}
\end{aligned}
$$

\section{An Example: Electric Field Imaging}

In this section we consider the case where the vector field that we want to recover is the electric field created by a static charge. There are many ways to recover the electric field from boundary data. However, here we use the electric field only to demonstrate our method. In order to avoid problems with singularities and to ensure that the field is band-limited, we place the source of the vector field outside the bounded 2-D area. In a real physical system, we do not expect to have to deal with real singularities anyway. The fields used here for reconstruction were selected only on the grounds of not containing singularities. No other selection criterion was used. One may find the reconstruction of more complicated fields, created by more than one point sources, in [21].

For the electric field recovery, the data, we relied entirely on, were voltage differences measured by pairs of sensors located along the border of the 2-D area. Such data measure the integral of the component of the electric field along the line that connects the two sensors. For the simulations we present here, these data were obtained by using Coulomb's law. It must be noted that the electric field is irrotational, so according to [18], only transversal measurements would be helpful to recover this field. However, the only realizable measurements for this application are longitudinal.

For our experiments, we employed the digital domain of Fig. 1 and we chose for parameter values: $2 U=11$ as domain size and $P=1$ as tile size. Hence, the domain consisted of 121 tiles and the number of the unknowns (the $E_{x}$ and $E_{y}$ components of the field for every tile of the domain) was 242. Four different cases for the location of the source of the vector field are reported. In the sampling process along line segments between sensors, so as to approximate the integral measurements by sums, we selected the sampling step to be equal to 1 (=tile size) in all cases. To exemplify the theory of the study described in this paper, we performed four sets of experiments.

In the first set of experiments, we used a data acquisition geometry that corresponded to uniformly sampled parameters $\rho$ and $\theta$, where the sampling criteria that we derived in Sect. 3 were satisfied. For the employed rectangle of interest, we have $\Delta x=\Delta y=P=1$ and $x_{\max }=U=5.5$. Hence, the sampling criteria of (45) and (46) yield:

$\Delta \rho \leq 0.7071$ and $\Delta \theta \leq 3.0512^{\circ}$

In order to meet these requirements, we selected for the sampling steps: $\Delta \rho=0.7$ and $\Delta \theta=3^{\circ}$. With the purpose of covering fully the region of interest (Fig. 1), the selected steps resulted in having 8 samples for the radial parameter and 120 samples for the angular parameter. As a result, the over-determined system of linear equations, the solution of which gave the reconstructed field, had $960(=8 \times 120)$ equations. We must note that we could have selected smaller sampling steps for $\Delta \rho$ and $\Delta \theta$. However, we opted out of this, because it would increase the number of equations too much and we would have to solve a prohibitively large system of linear equations. The solution of this system of equations was obtained by applying the least-squares method. The Householder orthogonalisation method [27], which is a numerically useful procedure in order to solve mean square value problems for cases where the condition number of the matrix of coefficients is large [28], was also tested for our reconstruction problem. However, the results we obtained were identical with the results we obtained using the leastsquares method. Moreover, it must be noted that since the residual we computed by using the least-squares method was not large when compared with the solution vector, there was no need to use the Cholesky method [4]. The reconstruction results are shown in Fig. 3(a). For the sake of comparison, Fig. 3(b) depicts the respective electric fields that were obtained by using directly the governing Coulomb's law.

In order to test the effectiveness of the sampling bounds that we propose in this paper, we ran three more sets of experiments without imposing the derived upper bounds for $\Delta \rho$ and $\Delta \theta$ in the sampling of the measurement geometry. More specifically, for the second set of experiments we chose $\Delta \rho=1$ and $\Delta \theta=2^{\circ}$. It is obvious, from (47), that such a selection for sampling step $\Delta \rho$ is a clear violation of the criterion we derived in Sect. 3 . The above choice for parameter values resulted in having 6 samples for the radial parameter and 180 samples for the angular parameter. Hence, the system of equations consisted of $1080(=6 \times 180)$ equations. In the third set of experiments, vector field recovery was carried out by using uniform sampling in the Radon domain and selecting for sampling steps $\Delta \rho=0.5$ 
(a) Recovered Field
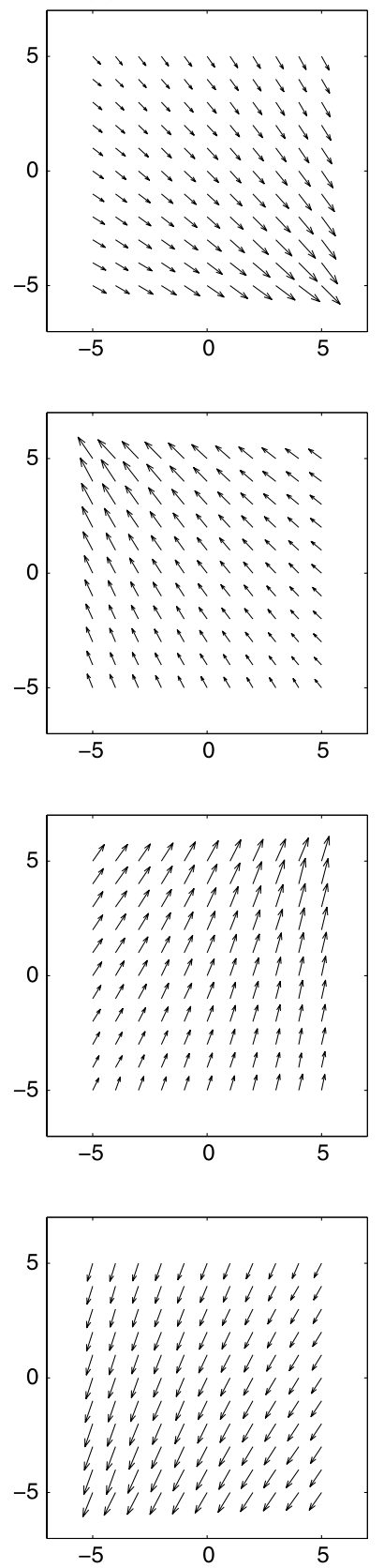

(b) Theoretical Field
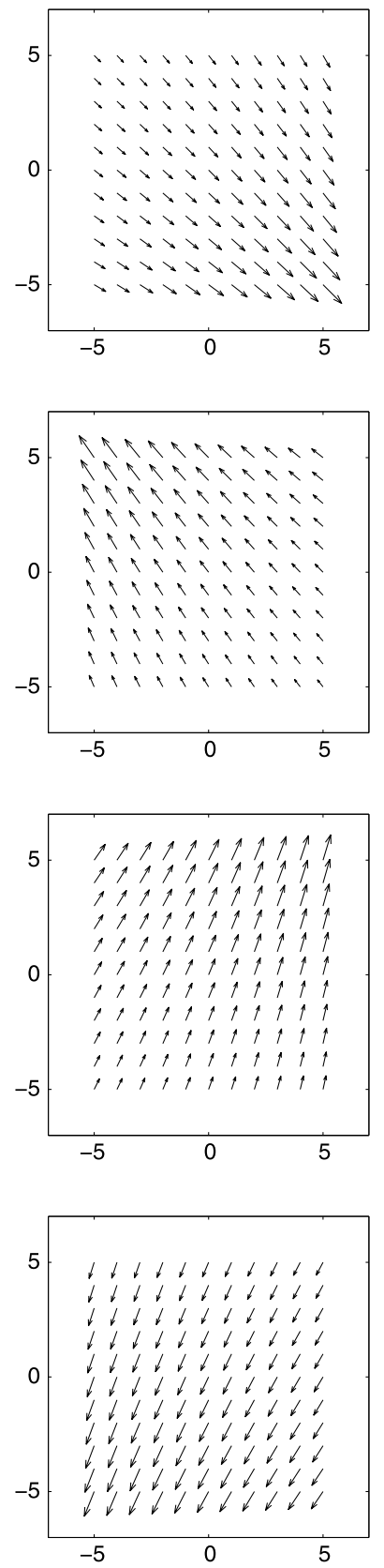

Fig. 3 Simulation results for the case when the proposed sampling criteria are met $\left(\Delta \rho=0.7\right.$ and $\left.\Delta \theta=3^{\circ}\right)$ and the location of the source of the electric field was (from top to bottom) at $(19,-19),(-16,21)$, $(12.5,30)$, and $(-19,-40)$ : (a) the recovered vector field; (b) the theoretical electric field as computed from Coulomb's law

and $\Delta \theta=4^{\circ}$. Hence, it is sampling step $\Delta \theta$, this time, the one that does not fulfil the sampling requirements worked out in this paper (equation (47)). This selection of parameter values resulted in having 11 samples for the radial parameter and 90 samples for the angular parameter. Hence, the linear system consisted of $990(=11 \times 90)$ equations. Finally, for the last set of experiments we chose $\Delta \rho=1$ and
$\Delta \theta=4^{\circ}$. Hence, both proposed criteria were not satisfied (equation (47)). This choice of sampling steps resulted in having 6 samples for the radial parameter and 90 samples for the angular parameter. Hence, the linear system consisted of $540(=6 \times 90)$ equations. It must be noted that the number of linear equations was about the same for the four sets of experiments, apart from the last one where it was inevitable to have a reduced number of linear equations. The relative magnitude reconstruction error plots (i.e., the plots of the absolute values of the differences between the magnitudes of the reconstructed fields and the theoretical ones divided by the theoretical magnitude) and the absolute angular reconstruction error plots (i.e., the plot of the absolute angular differences (in degrees) between the reconstructed vector field values and the theoretical ones) for the four sets of experiments can be seen in Fig. 4 and Fig. 5, respectively. We notice from these figures that the case where the derived sampling criteria were met outperforms the other three cases where we had a violation of these criteria, even when the number of equations used in the other cases was larger than the number of equations used when the sampling criteria were satisfied.

To appreciate better the degradation in the performance of the proposed algebraic reconstruction method by not imposing the upper sampling bounds for $\Delta \rho$ and $\Delta \theta$, in Fig. 6 and Fig. 7 we present the histograms of the errors in each case. By close examination of these figures, we may see that the violation of the lower bounds for the sampling rates of the radial and angular parameters resulted in having reconstructions of lower quality. In particular, it was found that the average difference in the vector field orientation measured in degrees was 35\% higher, when the upper bound on sampling interval $\Delta \theta$ was not imposed, as opposed to the case where both sampling criteria were met, whereas the average error in the magnitude was higher by $24 \%$. Similarly, it was found that the average difference in the vector field orientation measured in degrees was $24 \%$ higher, when the upper bound on sampling interval $\Delta \rho$ was not imposed, as opposed to the case where both sampling criteria were met, whereas the average error in the magnitude was higher by $10 \%$. The corresponding differences in the angular and magnitude errors for the case where both the lower bounds on sampling rates of parameters $\rho$ and $\theta$ were not imposed, over the case of fulfilling the derived upper sampling bounds, were $38 \%$ and $26 \%$, respectively.

\section{The Effect of the Sampling Rate on Resilience to Noise}

An important issue when solving inverse problems is the sensitivity of the solution to noise. In this section we investigate the effect of the sampling rate of the Radon domain 
Fig. 4 The relative magnitude error plots for the cases when: (a) the proposed sampling criteria were met $(\Delta \rho=0.7$ and $\left.\Delta \theta=3^{\circ}\right)$; (b) the proposed sampling criterion about the radial parameter was not fulfilled $\left(\Delta \rho=1\right.$ and $\Delta \theta=2^{\circ}$ ); (c) the proposed sampling criterion about the angular parameter was not fulfilled ( $\Delta \rho=0.5$ and $\Delta \theta=4^{\circ}$ ); (d) both proposed sampling criteria about the radial and angular parameters were violated $\left(\Delta \rho=1\right.$ and $\left.\Delta \theta=4^{\circ}\right)$. The location of the source of the electric field was (from top to bottom $)$ at $(19,-19),(-16,21)$, $(12.5,30)$, and $(-19,-40)$

Fig. 5 As in Fig. 4, but here the errors in vector field orientation are plotted

(a) Magn. I (\%)

(b) Magn. II (\%)

(c) Magn. III (\%)

(d) Magn. IV (\%)
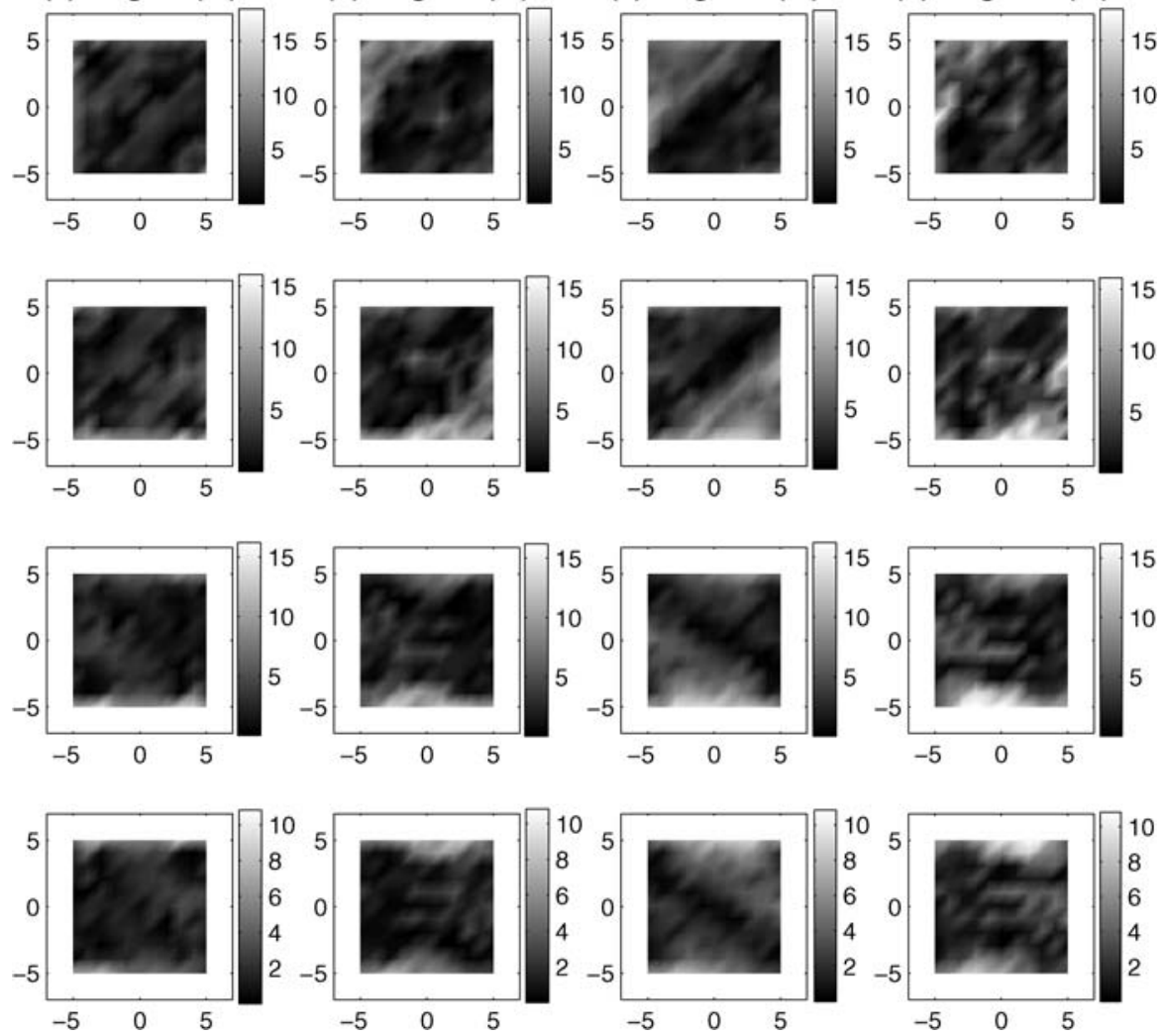

(a) Ang. $1\left({ }^{\circ}\right)$

(b) Ang. II ( $\left.{ }^{\circ}\right)$

(c) Ang. III ( $\left.{ }^{\circ}\right)$

(d) Ang. IV ( ${ }^{\circ}$ )
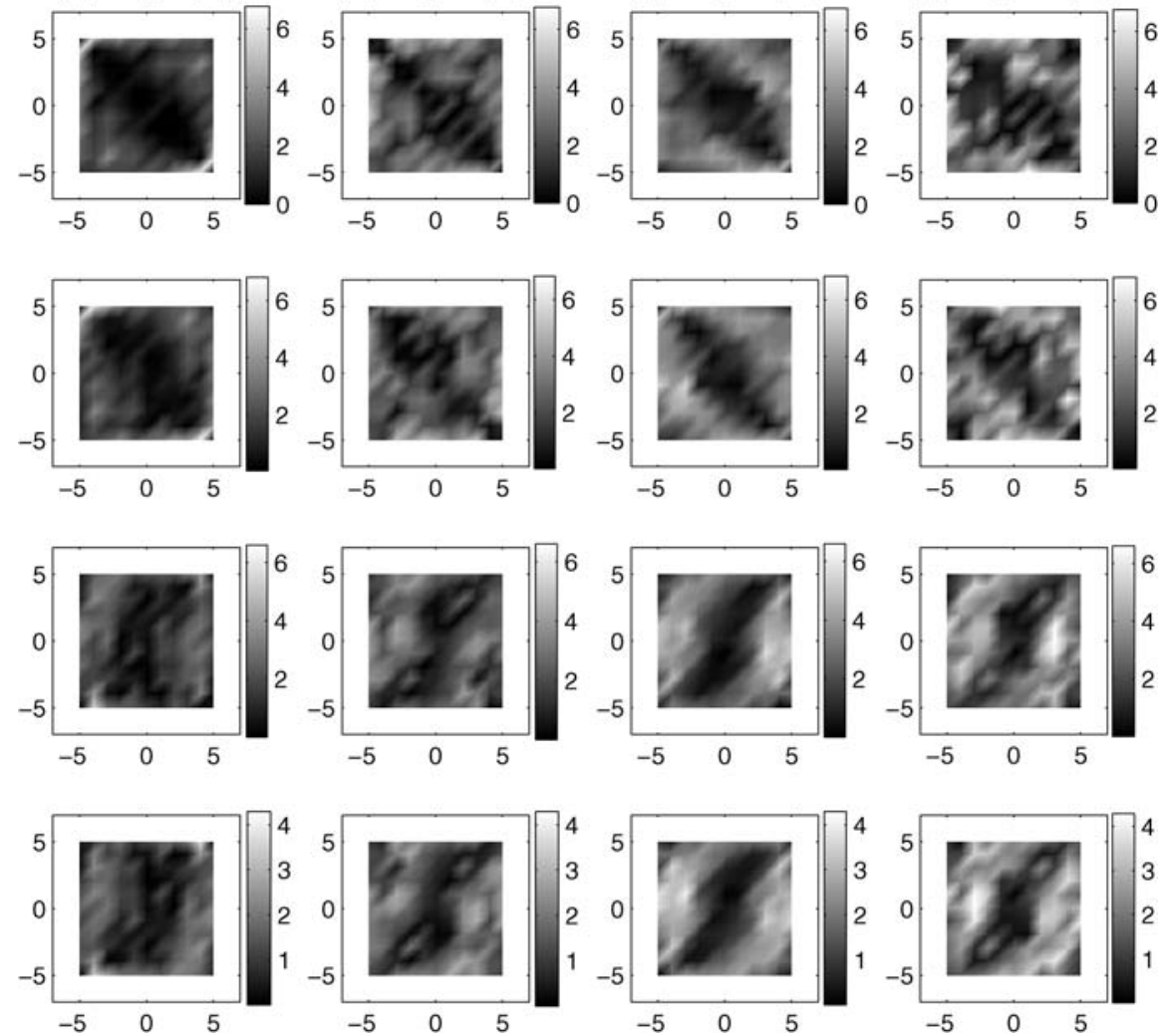
Fig. 6 The histograms of the relative magnitude errors for the cases when: the proposed sampling criteria were met $\left(\Delta \rho=0.7\right.$ and $\left.\Delta \theta=3^{\circ}\right)$ (left column); the proposed sampling criterion about the radial parameter was not fulfilled $\left(\Delta \rho=1\right.$ and $\left.\Delta \theta=2^{\circ}\right)$ (second column); the proposed sampling criterion about the angular parameter was not fulfilled $\left(\Delta \rho=0.5\right.$ and $\left.\Delta \theta=4^{\circ}\right)$ (third column); both proposed sampling criteria about the radial and angular parameters were violated $(\Delta \rho=1$ and $\Delta \theta=4^{\circ}$ ) (fourth column). The location of the source of the electric field was (from top to bottom $)$ at $(19,-19),(-16,21)$, $(12.5,30)$, and $(-19,-40)$. We note that the histograms of the last three columns have heavier tails towards higher values, when compared with the histograms of the first column
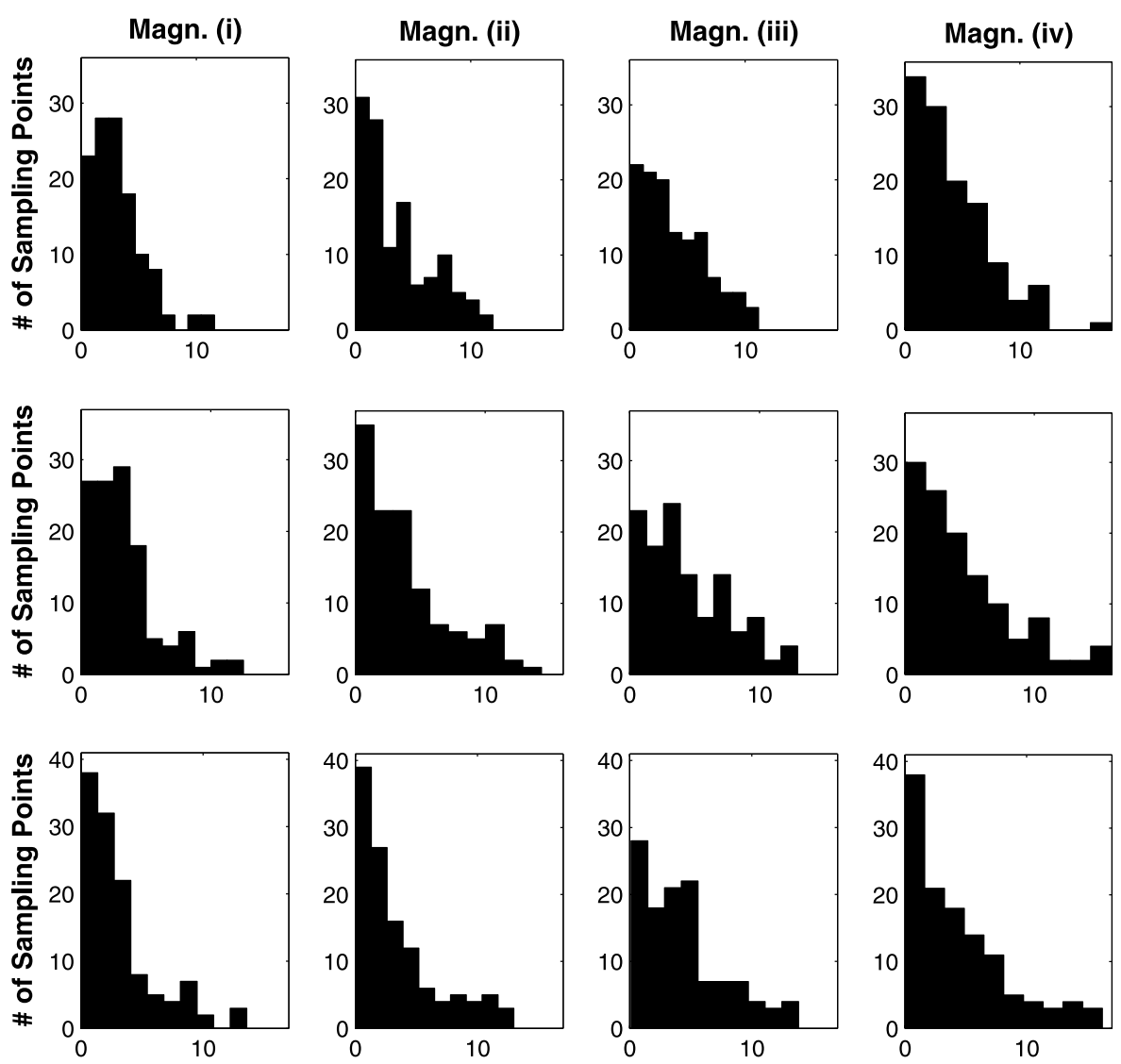

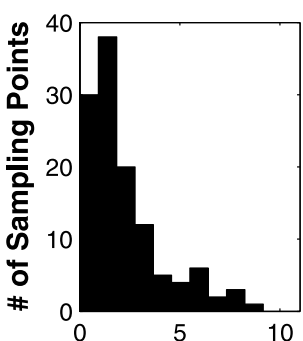

(\%)

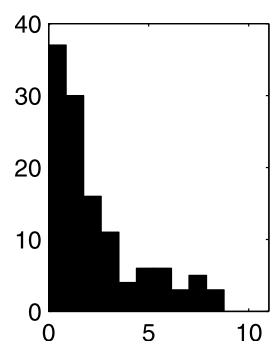

(\%)

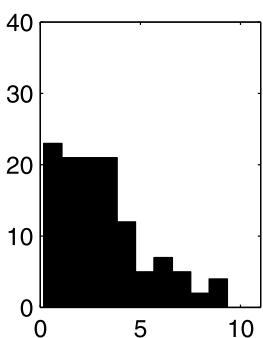

(\%)

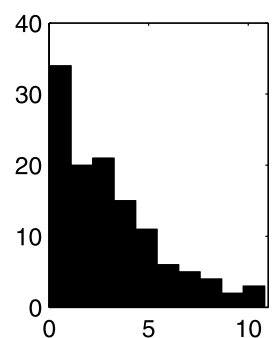

(\%) parameters on robustness against noise. In all experiments reported in the previous section, the measurement taken by each sensor was exactly the value predicted by Coulomb's law. In a practical system, however, some of the sensor measurements are expected to have inaccuracies. To emulate these effects, we considered the following:

(i) A noise value was added to a measurement as a fraction of the true value, with random sign. For example, $2 \%$ noise means that the sensor measurement was changed by $2 \%$ of the value dictated by Coulomb's law. The change was either incremental or decremental, the choice made at random for each sensor.

(ii) A sensor was moved away from its true position by a fraction of the true position. For example, if accord- ing to the theory, a sensor should be placed at position $(x, y)$, and we consider a $2 \%$ error, then, the coordinates of this sensor were shifted by $2 \%$ the corresponding correct values, with a positive or negative sign chosen at random.

(iii) Both the above errors were considered simultaneously.

We performed four series of experiments by perturbing, by the three types of noise described above, (a) only $25 \%$ of the sensors; (b) $50 \%$ of the sensors; (c) $75 \%$ of the sensors; (d) all sensors. In order to evaluate the robustness of the proposed sampling bounds against noise, we examined for each series of experiments, the four cases of Sect. 4: (a) when the proposed sampling criteria were met $(\Delta \rho=0.7$ and $\Delta \theta=3^{\circ}$ ); (b) when the proposed sampling criterion about 
Fig. 7 As in Fig. 6, but here the histograms of the errors in vector field orientation are plotted. Again, we note that the histograms of the last three columns have heavier tails towards higher values, when compared with the histograms of the first column
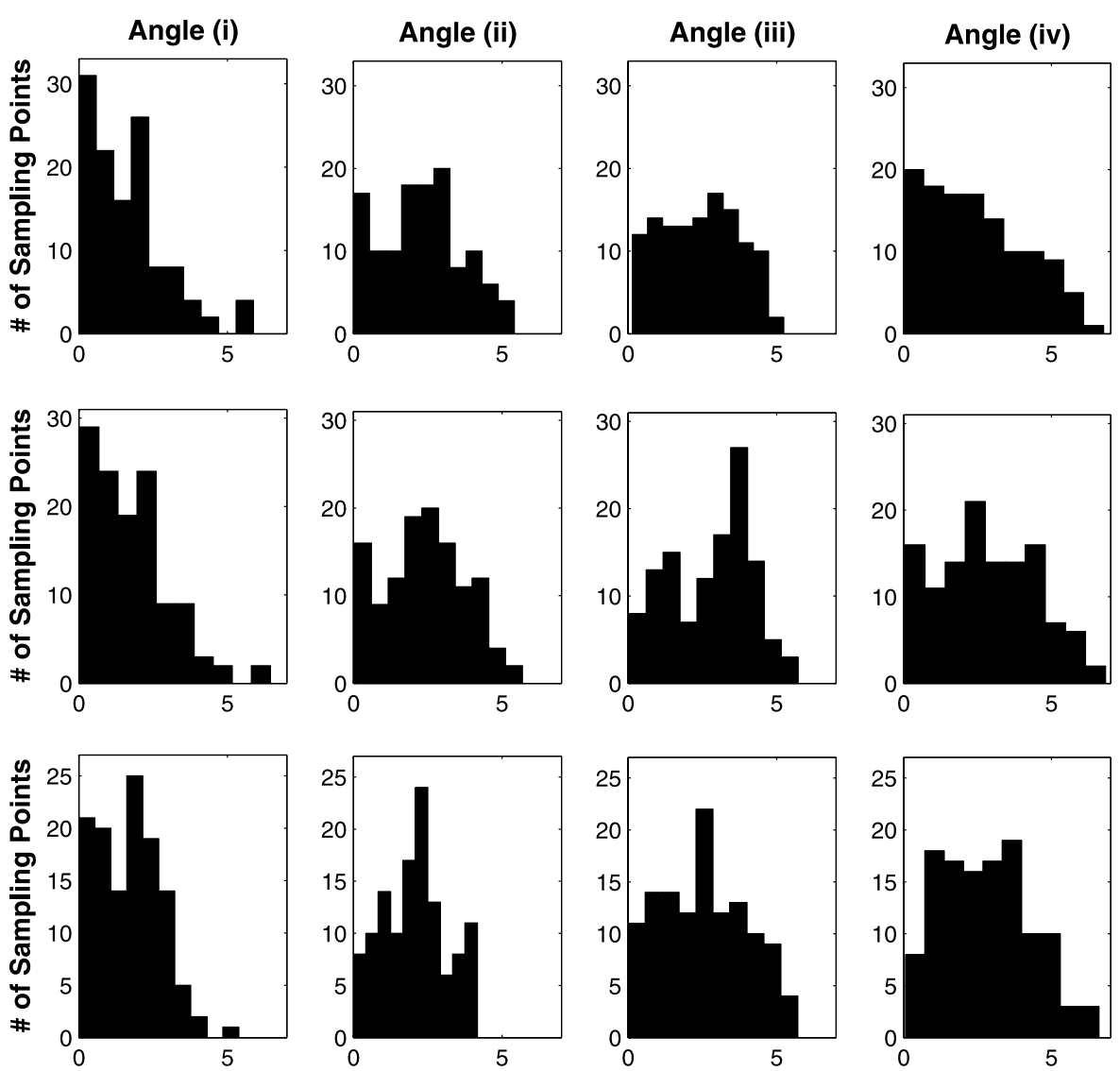

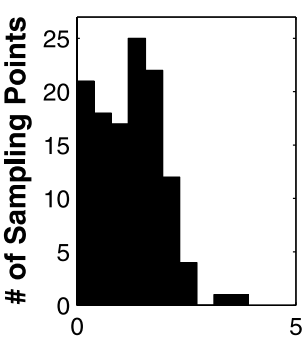

$\left({ }^{\circ}\right)$

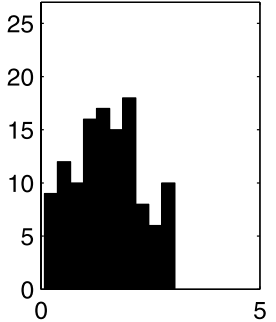

$\left({ }^{\circ}\right)$

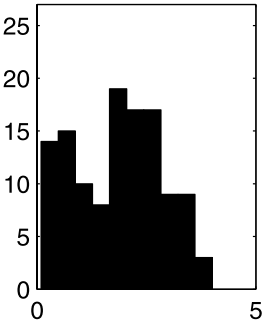

$\left({ }^{\circ}\right)$

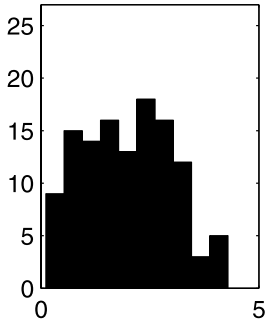

$\left({ }^{\circ}\right)$ the radial parameter was not fulfilled $(\Delta \rho=1$ and $\Delta \theta=2^{\circ}$ ); (c) when the proposed sampling criterion about the angular parameter was not fulfilled $(\Delta \rho=0.5$ and $\Delta \theta=4^{\circ}$ ); (d) when both proposed sampling criteria about the radial and angular parameters were violated. For every noise value (of each noise type, sampling rate and percentage of perturbed sensors), fifty simulations were performed and the average reconstruction errors in relative magnitude and absolute vector field orientation were obtained. The source for all the simulations was located at $(19,-19)$.

The results of these experiments are shown in Figs. 8-11. We observe that the employment of a measurement geometry that satisfies the sampling bounds, derived in Sect. 3, increases the resilience to all three types of noise, when compared with the cases where one or both of the proposed sampling criteria were not imposed.

\section{Discussion and Conclusions}

Vector field tomography is a field that has substantial potential for many applications. Solving, therefore, the inverse problem in vector field tomography has many advantages. The problem has been shown to be unsolvable in the continuous domain $[3,16,18]$. However, it has recently been demonstrated [21] that in the discrete domain, the recovery of all components of a 2-D band-limited vector field at the sampling points of a 2-D digitised bounded domain, based only on a discrete number of line-integral data, may be achieved.

Solving the reconstruction problem in the discrete domain requires the uniform sampling of the continuous Radon domain parameter space. In this paper we use sampling theory for deterministic band-limited signals to ad- 
Fig. 8 The comparison of the reconstruction performance in noisy environments for the four cases of sampling rates of Figs. 4-7: (a), (b) Errors in vector field orientation and magnitude, when noise was added to the measurements of $25 \%$ of the sensors, as a percentage of the true value. (c), (d) Errors in vector field orientation and magnitude, when small perturbations in the sensor positions were added. Position perturbations were a percentage of the true positions. (e), (f) Errors in vector field orientation and magnitude, when both sensors' measurements and positions were changed by a percentage of their true values. In all cases, $25 \%$ of the sensors were perturbed

Fig. 9 As in Fig. 8, but here $50 \%$ of the sensors were perturbed
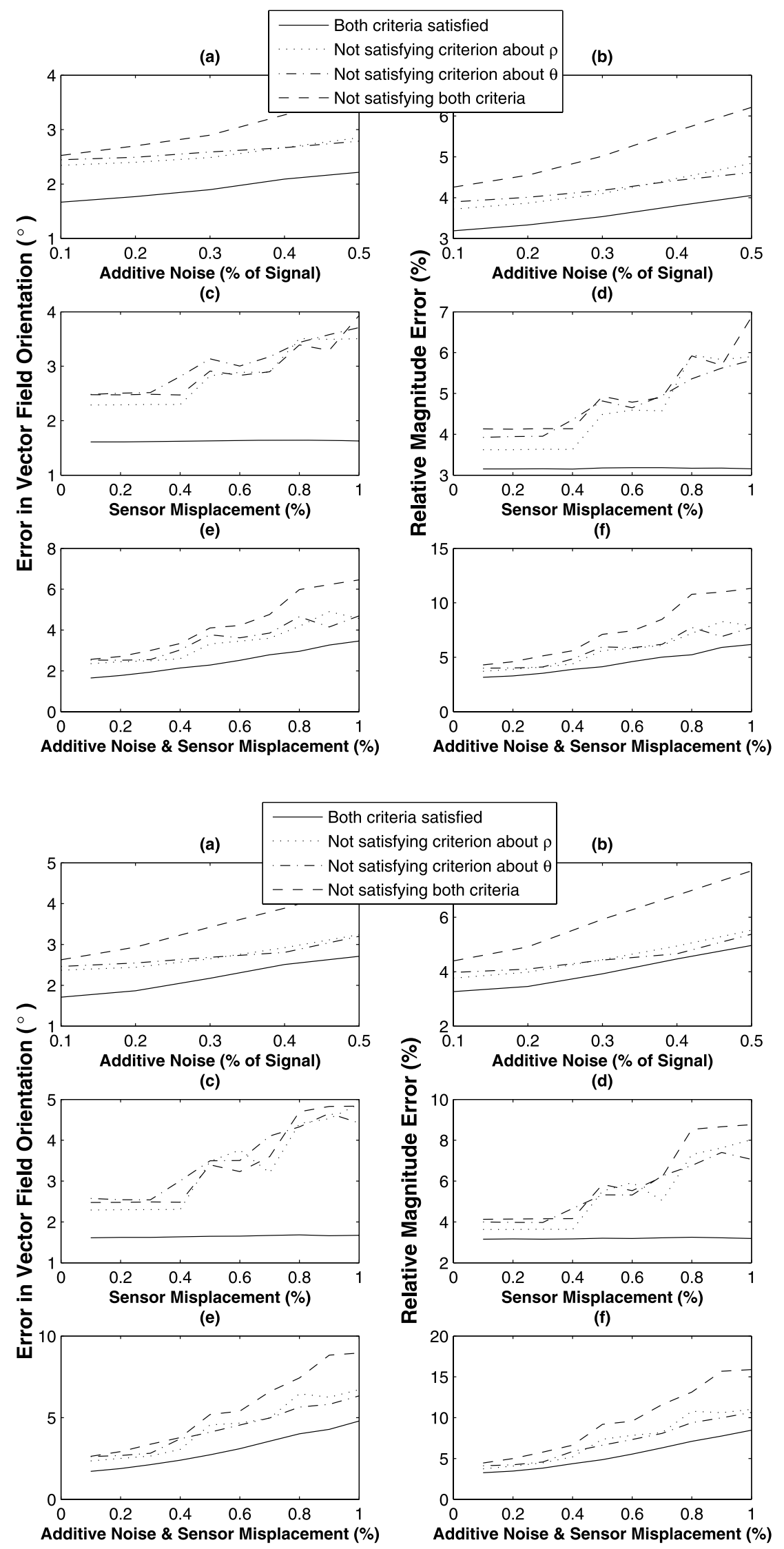
Fig. 10 As in Fig. 8, but here $75 \%$ of the sensors were perturbed

Fig. 11 As in Fig. 8, but here all sensors were perturbed
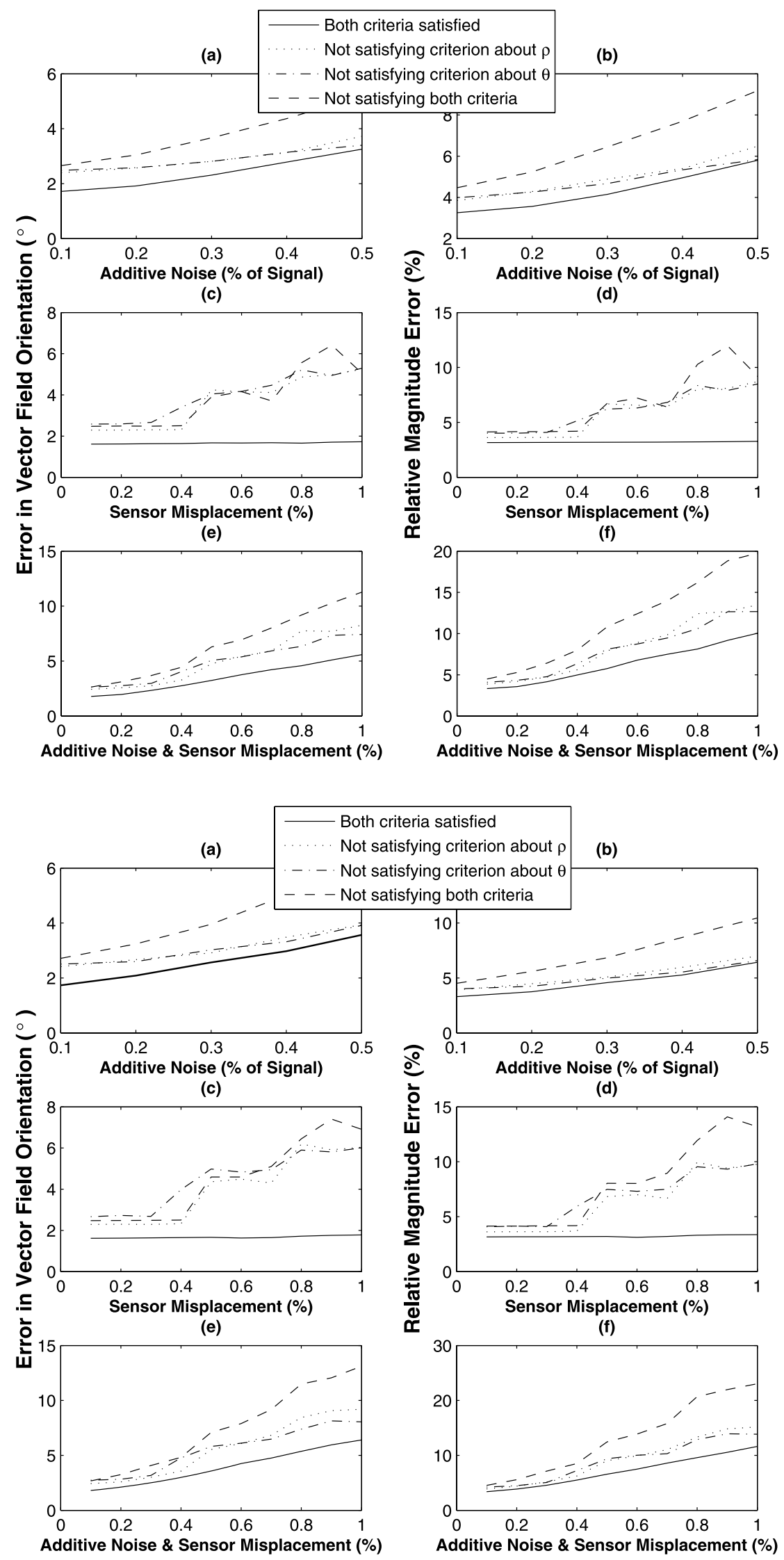
dress sampling issues. The sampling requirements, which must be imposed on the distances of the parameter domain variables in order not to lose boundary integral information, are derived. Evidence that shows the favourable behaviour of the proposed sampling bounds towards vector field reconstruction accuracy is provided by presenting an example. It is also shown that the implication of using a measurement geometry that violates the derived lower bounds is a degradation in the performance of the algebraic reconstruction method. This is expected, since, by not sampling the Radon parameters densely enough, the information content of the line-integral measurements is inadequate, and the aliasing problems that occur have an effect on the reconstruction quality.

An important issue when solving inverse problems is the sensitivity of the solution to noise. In the case of this problem, there are two possible sources of noise: inaccuracies in the sensor measurements and inaccuracies in the positions of the sensors. It is very encouraging, therefore, that more resilience to noise was observed when the sampling bounds, proposed in this paper, were imposed.

Acknowledgement This work was supported by the "Integrated Electronics" portfolio grant.

\section{References}

1. Aben, H.K.: Kerr effect tomography for general axisymmetric field. Appl. Opt. 26, 2921-2924 (1987)

2. Aben, H.K., Puro, A.: Photoelastic tomography for threedimensional flow birefringence studies. Inverse Probl. 13, 215221 (1997)

3. Braun, H., Hauck, A.: Tomographic reconstruction of vector fields. IEEE Trans. Signal Process. 39, 464-471 (1991)

4. Bronshtein, I.N., Semendyayev, K.A., Musiol, G., Muehlig, H.: Handbook of Mathematics, pp. 277-278. Springer, New York (2003)

5. Bukhgeim, A.A., Kazantsev, S.G.: Full reconstruction of a vector field from its attenuated vectorial Radon transform. In: Proceedings of the 22nd IASTED International Conference Modelling, Identification and Control, pp. 294-298 (2003)

6. Deans, S.R.: The Radon Transform and Some of Its Applications. Wiley, New York (1983)

7. Desbat, L., Wernsdorfer, A.: Direct algebraic reconstruction and optimal sampling in vector field tomography. IEEE Trans. Signal Process. 43, 1798-1808 (1995)

8. Efremov, N.P., Poluektov, N.P., Kharchenko, V.N.: Tomography of ion and atom velocities in plasmas. J. Quant. Spectrosc. Radiat. Transf. 53, 723-728 (1995)

9. Faris, G.W., Byer, R.L.: Three-dimensional beam-deflection optical tomography of a supersonic jet. Appl. Opt. 27, 5202-5212 (1988)

10. Hertz, H.M.: Kerr effect tomography for nonintrusive spatially resolved measurements of asymmetric electric field distributions. Appl. Opt. 25, 914-921 (1986)

11. Howe, B.M., Worcester, P.F., Spindel, R.C.: Ocean acoustic tomography: mesoscale velocity. J. Geophys. Res. 92, 3785-3806 (1987)
12. Johnson, S.A., Greenleaf, J.F., Tanaka, M., Flandro, G.: Reconstructing three-dimensional temperature and fluid velocity vector fields from acoustic transmission measurements. Instrum. Syst. Autom. Soc. Trans. 16, 3-15 (1977)

13. Juhlin, P.: Principles of Doppler tomography. LUTFD2/(TFMA92)/7002+17P, Lund Institute of Technology, Sweden, Dept. of Mathematics (1992)

14. Juhlin, P.: Doppler tomography. In: Proc. of the 15th Annu. Int. Conf. of the IEEE Eng. in Méd. and Biol. Soc. EMBC, pp. 212213 (1993)

15. Kramer, D.M., Lauterbur, P.C.: On the problem of reconstructing images of non-scalar parameters from projections. Applications to vector fields. IEEE Trans. Nucl. Sci. 26, 2674-2677 (1979)

16. Munk, W., Wunsch, C.: Observing the Ocean in the 1990s. Philos. Trans. R. Soc. Lond. Ser. A, Math. Phys. Sci. 307, 439-464 (1982)

17. Norton, S.J.: Tomographic reconstruction of 2-D vector fields: application to flow imaging. Geophys. J. Int. 97, 161-168 (1988)

18. Norton, S.J.: Unique tomographic reconstruction of vector fields using boundary data. IEEE Trans. Image Process. 1, 406-412 (1992)

19. Norton, S.J., Linzer, M.: Correcting for ray refraction in velocity and attenuation tomography: a perturbation approach. Ultrason. Imag. 4, 201-233 (1982)

20. Papoulis, A.: Probability, Random Variables, and Stochastic Processes, 2nd edn. McGraw-Hill, New York (1984)

21. Petrou, M., Giannakidis, A.: Complete tomographic reconstruction of 2-D vector fields using discrete integral data. Comput. J. (2010, to appear)

22. Prince, J.L.: Convolution backprojection formulae for 3D vector tomography with application to MRI. IEEE Trans. Image Process. 5, 1462-1472 (1996)

23. Rouseff, D., Winters, K.B.: Two-dimensional vector flow inversion by diffraction tomography. Inverse Probl. 10, 687-697 (1994)

24. Rouseff, D., Winters, K.B., Ewart, T.E.: Reconstruction of oceanic microstructure by tomography: a numerical feasibility study. J. Geophys. Res. 96, 8823-8833 (1991)

25. Sato, T., Aoki, H., Ikeda, O.: Introduction of mass conservation law to improve the tomographic estimation of flow-velocity distribution from differential time-of-flight data. J. Acoust. Soc. Am. 77, 2104-2106 (1985)

26. Schwarz, A.: Three-dimensional reconstruction of temperature and velocity fields in a furnace. In: Proc. of ECAPT, the Eur. Concert. Action on Process Tomogr., pp. 227-233 (1994)

27. Schwarz, H.R.: Numerische Mathematik. Teubner, Leipzig (1986)

28. Schwetlick, H., Kretzschmar, H.: Numerische Verfahren fur Naturwissenschaftler und Ingenieure. Fachbuchverlag, Leipzig (1991)

29. Segre, S.E.: The measurement of poloidal magnetic field in a Tokamak by the change of polarization of an electromagnetic wave. Plasma Phys. 20, 295-307 (1978)

30. Sharafutdinov, V.A.: Tomographic problem of photoelasticity. Proc. Soc. Photo-Opt. Instrum. Eng. 1843, 234-243 (1992)

31. Tao, Y.K., Davis, A.M., Izatt, J.A.: Single-pass volumetric bidirectional blood flow imaging spectral domain optical coherence tomography using a modified Hilbert transform. Opt. Express 16, 12350-12361 (2008)

32. Winters, K.B., Rouseff, D.: A filtered backprojection method for the tomographic reconstruction of fluid vorticity. Inverse Probl. 6, L33-L38 (1990)

33. Winters, K.B., Rouseff, D.: Tomographic reconstruction of stratified fluid flow. IEEE Trans. Ultrason. Ferroelectr. Freq. Control 40, 26-33 (1993)

34. Zahn, M.: Transform relationship between Kerr-effect optical phase shift and non-uniform electric field distributions. IEEE Trans. Dielectr. Electr. Insul. 1, 235-246 (1994) 


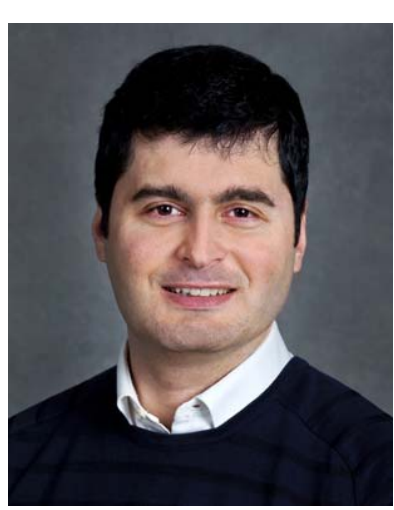

Archontis Giannakidis was born in Larisa, Greece, in 1976. He received a degree in Electrical \& Computer Engineering in 2000 from the Aristotle University of Thessaloniki, Thessaloniki, Greece. In 2003 he obtained an M.Sc. (Eng.) degree in Electronic and Electrical Engineering from the University of Leeds, Leeds, UK. Subsequently, he joined the Greek Army for 17 months as a Signals Corps 2nd Lieutenant in Reserve. In 2009 he received his Ph.D. degree in Electronic Engineering from the University of Surrey, Guildford, UK. Since February 2010, he has been working as a Postdoctoral Research Fellow with the Life Sciences Division, Lawrence Berkeley National Laboratory, Berkeley, CA, USA.

His research interests involve vector and tensor field tomographic reconstruction with application in medicine and biology. Areas of interest are radiographic imaging, magnetic resonance imaging (MRI), ultrasound imaging, and nuclear medicine imaging. Other interests include the quantitative analysis of diffusion tensor MRI heart data, the flow and diffusion modeling in porous media, the imaging of the conductivity distribution, and the study of lower-order statistics with application in biomedical signals. Dr. Giannakidis is a member of the Technical Chamber of Greece.

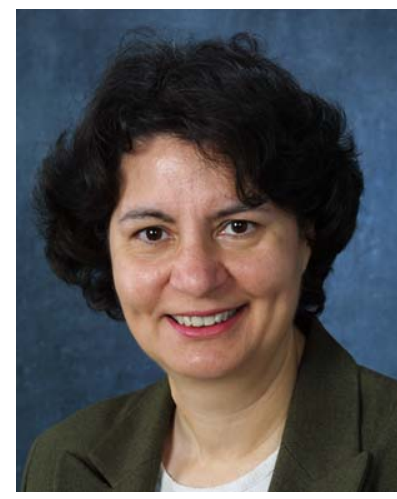

Maria Petrou studied Physics at the Aristotle University of Thessaloniki, Greece, Applied Mathematics in Cambridge and she did her $\mathrm{Ph} . \mathrm{D}$. in the Institute of Astronomy in Cambridge, UK. She obtained her D.Sc. also from Cambridge in 2009. She currently holds the Chair of Signal Processing at Imperial College London and is the Director of the Informatics and Telematics Institute of CERTH, Greece. She has published more than 350 scientific papers, on Astronomy, Remote Sensing, Computer Vision,

Machine Learning, Colour analysis, Industrial Inspection, and Medical Signal and Image Processing. She has co-authored two books "Image Processing: the fundamentals", first edition 1999, second edition 2010, and "Image Processing: dealing with texture", 2006, both published by John Wiley. She has also co-edited the book "Next generation artificial vision systems: reverse engineering the human visual system". She has supervised to successful completion $41 \mathrm{Ph} . \mathrm{D}$. theses. She is a Fellow of the Royal Academy of Engineering, Fellow of the City and Guilds Institute, Fellow of IET, Fellow of IAPR, Fellow of the Institute of Physics, Senior member of IEEE and a Distinguished Fellow of the British Machine Vision Association. She has served as a trustee of IET (2006-2009), as the IAPR newsletter editor (1994-1998) and as the IAPR treasurer (2002-2006). More information can be found in http://www.commsp.ee.ic.ac.uk/ mcpetrou. 